\title{
Recovery from Complex Regional Pain Syndrome after Super Refractory Status Epilepticus
}

\author{
Wonshik Kim, MD, Dayoung Kim, MD, Hyemi Lee, MD, Dong Wook Kim, MD \\ Department of Neurology, Konkuk University School of Medicine, Seoul, Korea
}

Received: May 14, 2019

Revised: September 2, 2019

Accepted: September 6, 2019

Corresponding author:

Dong Wook Kim, MD, PhD

Department of Neurology, Konkuk

University School of Medicine 120-1

Neungdong-ro, Gwangjin-gu, Seoul

05030, Korea

Tel: $+82-2-2030-7562$

Fax: +82-2-2030-7469

E-mail: drdongwkim@kuh.ac.kr

\section{Dear Editor}

Complex regional pain syndrome (CRPS) is a chronic pain condition characterized by spontaneous and evoked regional pain. CRPS can be differentiated from other chronic pain conditions by the presence of prominent autonomic and inflammatory changes in the region of pain. The exact pathogenesis of CRPS is still unclear, but there seems to be involvement of multiple peripheral and central mechanisms, including peripheral nerve injury, inflammation, neuronal plasticity, and autonomic dysregulation. The relative contributions of these different mechanisms probably differ from one individual patient to another and even within a patient over time. ${ }^{1}$

Although acute CRPS may resolve with conservative medical care, treatment of chronic CRPS is mostly difficult, and there's need for comprehensive multidisciplinary treatment, such as drug treatment, local injection to sympathetic ganglion, spinal cord stimulation, psychological and physical treatment. In intractable CRPS patients, other non-traditional therapeutic approaches such as plasma exchange, medical marijuana, and electroconvulsive therapy (ECT) have been tested. ${ }^{2}$ We recently encountered a patient who experienced transient, but complete recovered from CRPS after super refractory status epilepticus.

The patient, 55-year-old man was transferred to our hospital with generalized tonic-clonic seizure that lasted for more than 15 minutes. A former construction worker, he became disabled six years ago with multiple fracture in the ribs and the right fibula after falling from a height of three meters. Since the injury, he suffered persistent pain on the back and leg despite the fracture being healed. $\mathrm{He}$ described spontaneous, excruciating pain-mainly burning and deep aching-in his back and right lower leg, as well as allodynia and hyperalgesia. A three-phase bone scan showed signs associated with CRPS. He tried several pain treatments, including several opioids, antiepileptic drugs, antidepressants, lumbar epidural block, regional sympathetic nerve blocks, transcutaneous nerve stimulation as well as acupuncture. All these treatments had an insufficient effect. At the time of admission, the patient was comatose, and did not responded to painful stimuli. He was receiving duloxetine 60 $\mathrm{mg}$, pregabalin $600 \mathrm{mg}$, oxcarbazepine $600 \mathrm{mg}$, tapentadol $400 \mathrm{mg}$, clonazepam $4.5 \mathrm{mg}$, oxycodone $80 \mathrm{mg}$, and trazodone $100 \mathrm{mg}$. Initial brain MRI including diffusion-weighted image showed no abnormality, but EEG, which was performed at two hours after the admission, showed continuous 2-Hz spike and waves in both frontal areas (Fig. 1), which lasted for three days and terminated with

This is an Open Access article distributed under the terms of the Creative Commons Attribution Non-Commercial License (http://creativecommons.org/licenses/by-nc/4.0/) which permits unrestricted non-commercial use, distribution, and reproduction in any medium, provided the original work is properly cited.

Copyright (C) 2019 Korean Clinical Epilepsy Research Society 


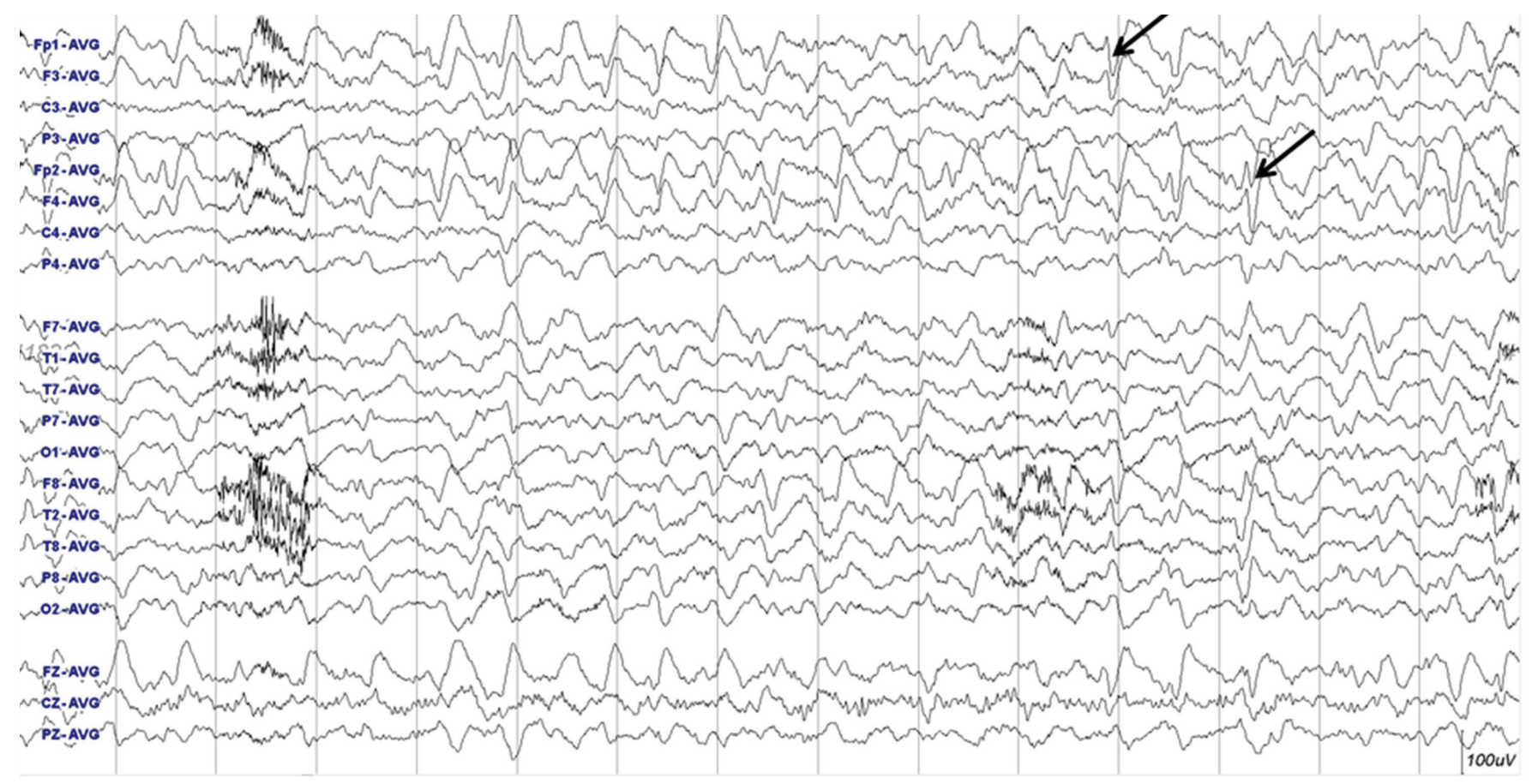

Fig. 1. EEG showed continuous 2-Hz spike and waves in both frontal areas (arrows).

the continuous infusion of midazolam coupled with the treatment of several antiepileptic drugs (lacosamide $400 \mathrm{mg}$, levetiracetam $3,000 \mathrm{mg}$, topiramate $400 \mathrm{mg}$, valproic acid 1,200 mg, and perampanel $10 \mathrm{mg}$ ). He gradually recovered after five days' coma therapy, and fully recovered two weeks after admission. Surprisingly, he reported no pain after recovering from status epilepticus, and only complained of short-term memory loss. He was well maintained with lacosamide $400 \mathrm{mg}$, levetiracetam $1,000 \mathrm{mg}$, and perampanel $6 \mathrm{mg}$. However, the pain recurred three weeks after discharge and gradually got worse, and returned to previous state seven weeks after discharge.

It has long been revealed that nociception threshold is high in epilepsy patients, and monoamine-mediated modulation of central pain inhibitory system is responsible for the ictal and post-ictal analgesia in these patients. ${ }^{4}$ Although central pain modulation is an important mechanism of pathogenesis in chronic CRPS, there is limited information on the analgesic role of seizure or status epilepticus in CRPS patients. We only found several reports on the beneficial effects of 'convulsion' of ECT on CRPS patients. ${ }^{2}$ These reports indicated that repeated ECT could relieve pain in CRPS, and reduction could last for several weeks as was the case with our patient. ${ }^{2}$ It is possible that the effect of ECT on CRPS may be due to the beneficial effect of ECT on the co-morbid depression in CRPS patients, but ECT has been used in the treatment of pain such as phantom limb pain and somatic pain as well as CRPS for over 50 years. ${ }^{4}$ Therefore, it can be concluded that the transient, but complete recovery from pain in our patient resulted from the neuromodulation effect of prolonged status epilepticus, and it did not last for more than several weeks as in ECT therapy. In addition, the temporary improvement of CRPS may be in part attributable to the treatment of midazolam or other antiepileptic drugs, because there are reports of the effects of ketamine, another widely used antiepileptic drug for status epilepticus, on the CRPS. ${ }^{5}$

In summary, we herein report the first patient who experienced transient, but complete recovery from CRPS after prolonged status epilepticus. The mechanism of the pain-relieving effect of status epilepticus was unknown, but it would be similar to that of ECT on chronic pain, the seizure-related modulation of central pain inhibitory system.

\section{Notes}

\section{Conflicts of interest}

No potential conflicts of interest relevant to this article was reported. 


\section{Author contributions}

Conceptualization: DWK. Data curation: HL, DK, WK. Formal analysis: HL, DWK. Methodology: DWK. Supervision: DWK. Writing—original draft: HL, DK. Writing — review \& editing: DWK.

\section{Acknowledgments}

None.

\section{References}

1. Urits I, Shen AH, Jones MR, Viswanath O, Kaye AD. Complex regional pain syndrome, current concepts and treatment op- tions. Curr Pain Headache Rep 2018;22:10.

2. Wolanin MW, Gulevski V, Schwartzman RJ. Treatment of CRPS with ECT. Pain Physician 2007;10:573-578.

3. Freitas RL, Ferreira CM, Ribeiro SJ, et al. Intrinsic neural circuits between dorsal midbrain neurons that control fear-induced responses and seizure activity and nuclei of the pain inhibitory system elaborating postictal antinociceptive processes: A functional neuroanatomical and neuropharmacological study. Exp Neurol 2005; 191:225-242.

4. Leong K, Tham JC, Scamvougeras A, Vila-Rodriguez F. Electroconvulsive therapy treatment in patients with somatic symptom and related disorders. Neuropsychiatr Dis Treat 2015;11:25652572.

5. Niesters M, Martinin C, Dahan A. Ketamine for chronic pain: risks and benefits. Br J Clin Pharmacol 2014;77:357-367. 